\title{
Soft lithography for micro- and nanoscale patterning
}

\author{
Dong Qin ${ }^{1}$, Younan $\mathrm{Xia}^{2} \&$ George M Whitesides ${ }^{3}$
}

\begin{abstract}
${ }^{1}$ Nano Research Facility and Department of Energy, Environmental, and Chemical Engineering and ${ }^{2}$ Department of Biomedical Engineering, Washington University, St. Louis, Missouri, USA. ${ }^{3}$ Department of Chemistry and Chemical Biology, Harvard University, Cambridge, Massachusetts, USA. Correspondence should be addressed to G.M.W. (gmwhitesides@gmwgroup.harvard.edu).
\end{abstract}

Published online 18 February 2010; doi:10.1038/nprot.2009.234

\begin{abstract}
This protocol provides an introduction to soft lithography-a collection of techniques based on printing, molding and embossing with an elastomeric stamp. Soft lithography provides access to three-dimensional and curved structures, tolerates a wide variety of materials, generates well-defined and controllable surface chemistries, and is generally compatible with biological applications. It is also low in cost, experimentally convenient and has emerged as a technology useful for a number of applications that include cell biology, microfluidics, lab-on-a-chip, microelectromechanical systems and flexible electronics/photonics. As examples, here we focus on three of the commonly used soft lithographic techniques: (i) microcontact printing of alkanethiols and proteins on gold-coated and glass substrates; (ii) replica molding for fabrication of microfluidic devices in poly(dimethyl siloxane), and of nanostructures in polyurethane or epoxy; and (iii) solvent-assisted micromolding of nanostructures in poly (methyl methacrylate).
\end{abstract}

\section{INTRODUCTION}

The strategy of 'smaller brings new capability' has begun to change the world of biotechnology as it has transformed microelectronics ${ }^{1}$. Successful applications of small systems include microarrays for high-speed DNA sequencing ${ }^{2}$, microfluidic devices for performing $\mathrm{PCR}^{3}$, lab-on-a-chip (LOC) systems for synthesis and analysis of peptide and oligonucleotide libraries ${ }^{4}$, and microchips for drug screening ${ }^{5}$ and for investigation of cultured cells ${ }^{6}$. As the workhorse of microfabrication, photolithography ${ }^{7}$ has contributed an important role in most of these applications, including the fabrication of DNA arrays in the late 1980s (ref. 8). However, this technique has a number of limitations for applications related to biological systems. First, photolithography is an intrinsically expensive process because the equipment used was developed for the highly demanding processes required for fabrication of microelectronic devices. The capital investment required to build a clean room makes photolithography less than accessible to most chemists, biochemists and biologists. Thus, photolithography is most successful when applied to a limited set of materials. Users wishing to study 'dirty' organic systems are usually excluded from an electronics-qualified clean-room facility. Second, photolithography is often carried out by projecting a pattern on a photomask onto a photoresist film. Although photomasks are commercially available, the time and cost involved in the fabrication of such masks present a significant barrier to the use of photolithography in rapid, inexpensive prototyping of test patterns and devices. Third, photolithography provides little or no control over surface chemistry, and it is not applicable to curved or non-planar substrates.

Soft lithography ${ }^{9}$ represents a conceptually different approach to rapid prototyping of various types of both microscale and nanoscale structures, and devices on planar, curved, flexible and soft substrates especially when low cost is required. A large number of patterning techniques - microcontact printing $(\mu \mathrm{CP})^{10}$, replica molding $(\mathrm{REM})^{11}$, microtransfer molding ${ }^{12}$, micromolding in capillary $^{13}$, solvent-assisted micromolding (SAMIM) ${ }^{14}$, phaseshifting edge lithography ${ }^{15}$, nanotransfer printing ${ }^{16}$, decal transfer lithography ${ }^{17}$ and nanoskiving ${ }^{18}$ _ form the basis of soft lithography; they are essentially based on printing, molding and embossing with an elastomeric stamp (Protocols for $\mu \mathrm{CP}$, REM and SAMIM are described in the Procedure and outlined in Fig. 1). New variants such as dip-pen nanolithography have also emerged ${ }^{19-21}$. All these techniques use organic and polymeric materials that are referred to as soft matter by physicists.

\section{$\mu \mathrm{CP}$}

As the forerunner of soft lithography, $\mu \mathrm{CP}$ provides an attractive route to microscale patterns and structures needed for applications in biotechnology. For example, $\mu \mathrm{CP}$ offers an ability to engineer the properties of a surface with molecular-level detail using selfassembled monolayers (SAMs) of alkanethiols on a substrate coated with a metal such as gold $(\mathrm{Au})$, silver $(\mathrm{Ag})$, copper $(\mathrm{Cu})$, palladium $(\mathrm{Pd})$ and platinum $(\mathrm{Pt})^{22}$. In this study, the substrate refers to a physical object, which is somewhat different from the meaning in enzymology. Typical planar substrates for forming alkanethiolate SAMs include thin films of a metal deposited on silicon, mica, glass or even plastic materials. The substrates can be easily prepared by physical vapor deposition methods, such as thermal or electron beam evaporation ${ }^{23}$. The patterned SAMs have proved valuable for studying the role of spatial signaling in cell biology by exquisitely controlling the molecular structure of a surface in contact with cells ${ }^{24}$. One can create well-defined regions with 'cell-friendly' (proteincovered) and 'cell-unfriendly' (polyethylene glycol-terminated) SAMs to pattern endothelial cells on a surface and then examine how the cells respond to lateral confinement, and to being released from confinement $^{24}$. Furthermore, $\mu \mathrm{CP}$ has been successfully used to print precise patterns of axon guidance molecules, which can then serve as templates for growing chick retinal ganglion cell axons ${ }^{25}$.

\section{REM}

Alternatively, REM provides a new method for fabricating microfluidic devices using poly(dimethyl siloxane) (PDMS) ${ }^{26}$, and this method has proved to be particularly suitable for various biomedical applications ${ }^{27-32}$. Compared with the inorganic materials such as silicon and glass that are used in microelectronics and optics, and which have been explored but largely discarded for 
biologically directed microfluidics, PDMS offers a number of unique and attractive features. They $\operatorname{are}^{33}$ : (i) It has a shear modulus of $0.25 \mathrm{MPa}$ and a Young's modulus of roughly $0.5 \mathrm{MPa}$ (characteristic of a moderately stiff elastomer). This elastomeric character allows it to conform to a surface and achieve atomic-level contact, a feature that is useful in forming and in sealing microfluidic systems. (ii) It is non-toxic and readily available from commercial sources at acceptable prices $(\sim 80 / \mathrm{kg})$. (iii) It is optically transparent down to about $300 \mathrm{~nm}$. (iv) It is intrinsically hydrophobic (with a water contact angle of $\sim 110^{\circ}$ ), but its surface can be modified by brief exposure to an oxygen plasma to become hydrophilic (with a water contact angle around $10^{\circ}$ ). (v) It can adhere and seal reversibly or, after oxidation, irreversibly to many different types of substrates ${ }^{26,34}$. The ease of production associated with PDMS microfluidic structures will become even greater as the focus of microfluidics shifts from demonstration of components and devices to development of fully functional systems $s^{31,35,36}$.

Soft lithography also provides a set of attractive tools for nanofabrication. 'Nanofabrication' refers to a process that makes functional structures with at least one lateral dimension smaller than $100 \mathrm{~nm}$ (ref. 37). REM and SAMIM are two techniques based on molding or embossing with an elastomeric stamp. Both can produce nanoscale structures in various soft materials, in parallel, over large areas $\left(3 \mathrm{~cm}^{2}\right.$ with $100-\mathrm{nm}$ features $)$, and at relatively low cost. When combined with selective etching and lift-off, these nanostructures have been transferred into metals and then used as substrates for biosensing. The methods of detection have included optical diffraction $^{38}$, surface plasmon resonance ${ }^{39}$, surface-enhanced Raman scattering ${ }^{40}$ and electrochemistry ${ }^{41}$.

\section{Photolithography versus soft lithography}

Photolithography will continue as the dominant technology in fabrication of semiconductor devices and systems that have stringent requirements on the alignment, continuity, isolation and uniformity in the final patterns. There are, however, many existing and emerging applications for soft lithography that take advantage of (or require) the characteristics of these techniques. For example, soft lithography offers access to a broader range of materials, as well as experimental simplicity and flexibility in forming certain types of test patterns. Procedures involving relatively large features (such as those used in microfluidic devices) can be conducted in an ordinary chemical laboratory and are thus particularly useful to those who do not have routine access to clean room facilities or for applications where the fabrication cost is a serious concern. At the current stage of development, soft lithography still relies on the use of photolithography to generate the master. Once the master is available, most of the fabrication tasks can be continued outside a clean room with the use of only a printing or molding procedure. As services for preparing the master are available from a number of sites through the NSF-supported National Nanotechnology Infrastructure Network (NNIN), there is essentially no need to build and maintain a clean room facility before one can start to explore the use of microscale structures, devices and systems.

\section{Experimental design}

Figure 1 outlines the four major steps of the procedure generally used for soft lithography: (i) pattern design, (ii) fabrication of the mask and then the master, (iii) fabrication of the PDMS stamp and (iv) fabrication of micro- and nanostructures with the stamp by
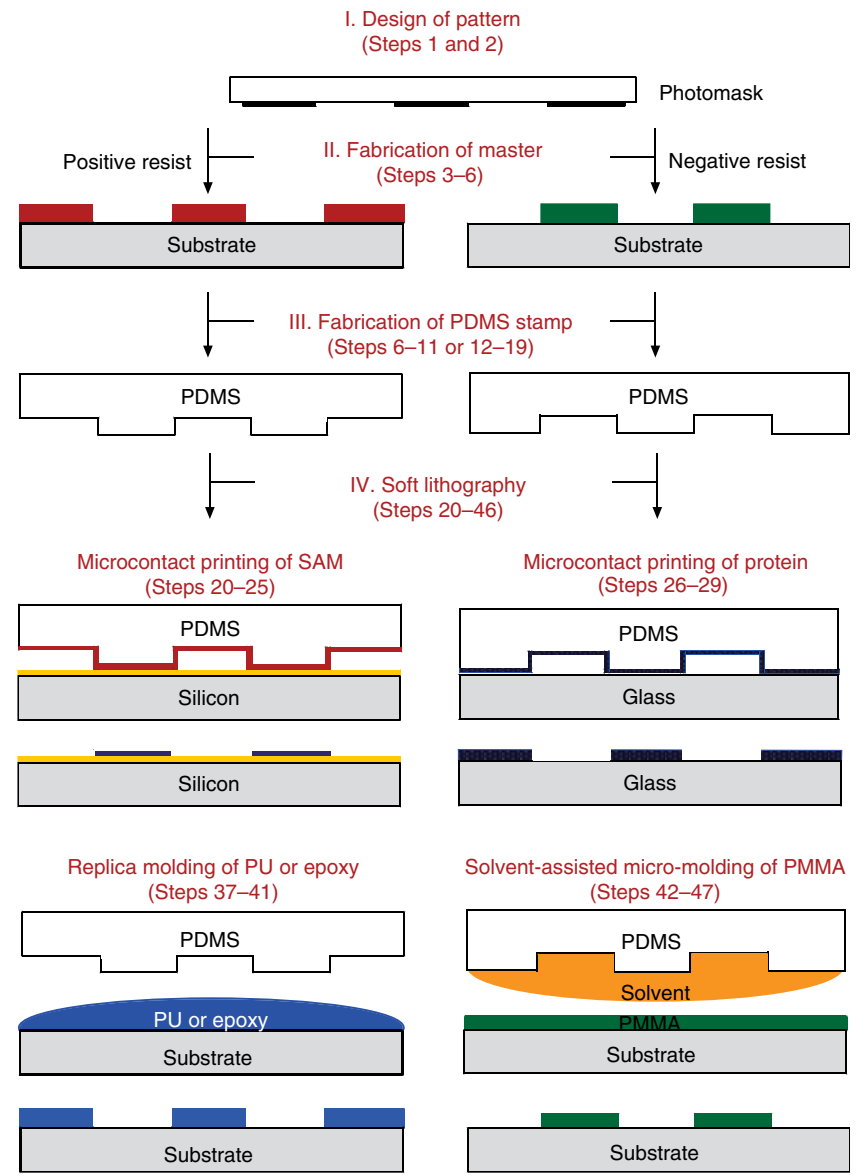

Solvent-assisted micro-molding of PMMA (Steps 42-47)

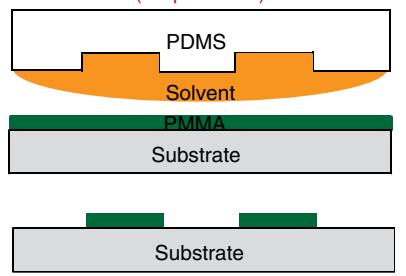

Figure 1 | Schematic illustration of the four major steps involved in soft lithography and three major soft lithographic techniques.

printing, molding and embossing. The term soft lithography 'refers to the fabrication of patterned copies using the PDMS stamp'; and the overall process - from pattern design to fabrication of functional structures-is sometimes (when the convenience of the method for rapid generation of functional structures is the feature emphasized) referred to as 'rapid prototyping'42.

Design of pattern. A number of computer-aided design (CAD) software programs are available to design a pattern. Adobe Freehand and Illustrator are two vector-based drawing programs well suited for quick design of a pattern with feature sizes down to $1 \mu \mathrm{m}$. These two software packages are widely accessible, easy to learn and particularly useful for fabricating photomasks on transparency films. Autodesk AutoCAD and DesignCAD are two advanced tools with more capabilities such as higher precision and higher drawing speed and are commonly used by a commercial supplier to design the pattern on a chrome mask-a quartz (or fused silica) plate patterned with opaque chrome on its surface.

Fabrication of mask. High-resolution printing provides a simple and convenient method for fabricating photomasks on transparency films ${ }^{42}$. The pattern created using Freehand or Illustrator and saved as an EPS file is compatible with most of the conventional printers or writers. Typically, a high-resolution commercial printer (5,060 d.p.i., with a dot size of $5 \mu \mathrm{m})$ can generate lines as thin as $20 \mu \mathrm{m}$ and with acceptable edge resolution; this size range 
is appropriate for most microfluidic applications. For higher resolutions down to $1 \mu \mathrm{m}$ or submicron, chrome photomasks are typically required.

High-resolution printing service is available from the publication departments of universities or large companies, or from local commercial printing shops. CAD/ART services in California offer 20,000 d.p.i. printouts. The cost is substantially less expensive than chrome masks. Chrome photomasks are available to be ordered from commercial suppliers such as Photo Sciences in the price range from hundreds to thousands of dollars depending on the feature size and density of patterns on a photomask. For users in the United States, services that prepare chrome masks are accessible at a number of sites through the NSF-supported NNIN. For proof of concept and survey experiments, transparency masks are highly recommended to save cost and improve efficiency.

Fabrication of master. Photolithography and e-beam lithography (EBL) are the primary two techniques for fabricating a 'master' that contains patterned relief structures on the surface. However, other relief structures such as diffraction gratings and metal grids for electron microscopy can also be useful for the fabrication of masters with certain simple patterns ${ }^{43}$. Conventional photolithography is a classic technique for patterning structures with feature sizes larger than $1 \mu \mathrm{m}$ (ref. 7). Photolithography is often conducted in a clean room that is largely developed and used by the semiconductor industry to manufacture microelectronic chips. In academia, a clean room is usually located in a facility associated with an engineering program. Every user of a clean room needs to go through an extensive training session before he/she can access the facility. The primary advantage of EBL over photolithography is its capacity to generate structures (20-30 $\mathrm{nm}$ in lateral dimensions) much smaller than those available through photolithography $(1-2 \mu \mathrm{m})$, which is mainly limited by optical diffraction (Cornell NanoScale Science \& Technology Facility). EBL starts from design and creation of the desired pattern as a $2 \mathrm{D}$ vector graphics-that is, use of geometrical primitives such as points, lines, curves and polygons to represent the pattern. As such, EBL is a serial process: it is generally much slower than photolithography, more expensive and subject to much longer waits for access to the facility. In general, EBL is a process that requires expensive equipment and an experienced operator. For users in the United States, access to both photolithography and EBL is also available through the NNIN.

Fabrication of elastomeric stamp. The key element of soft lithography is an elastomeric stamp with patterns as relief structures on its surface. The stamp is typically fabricated by casting a liquid precursor against a master whose surface has been patterned with the complementary structures. The mechanical properties of an elastomeric stamp are critical to its ability to transfer a pattern with high fidelity. In principle, any elastomer can be used to cast the stamp although most work has focused on the silicone-based rubber or cross-linked PDMS. Among various types of commercial PDMS, Sylgard 184 from Dow Corning has been most commonly used for fabrication of stamps with feature sizes larger than $500 \mathrm{~nm}$. Composite stamps consisting of two layers-a stiff layer (30- to $40-\mu \mathrm{m}$-thick h-PDMS) supported by a flexible layer (3- to 5-mm thick Sylgard 184 PDMS) — can extend the capability of soft lithography down to the $50-100 \mathrm{~nm}$ regime $^{44}$. Figure 2 outlines a procedure for fabricating Sylgard 184 and h-/184 PDMS composite

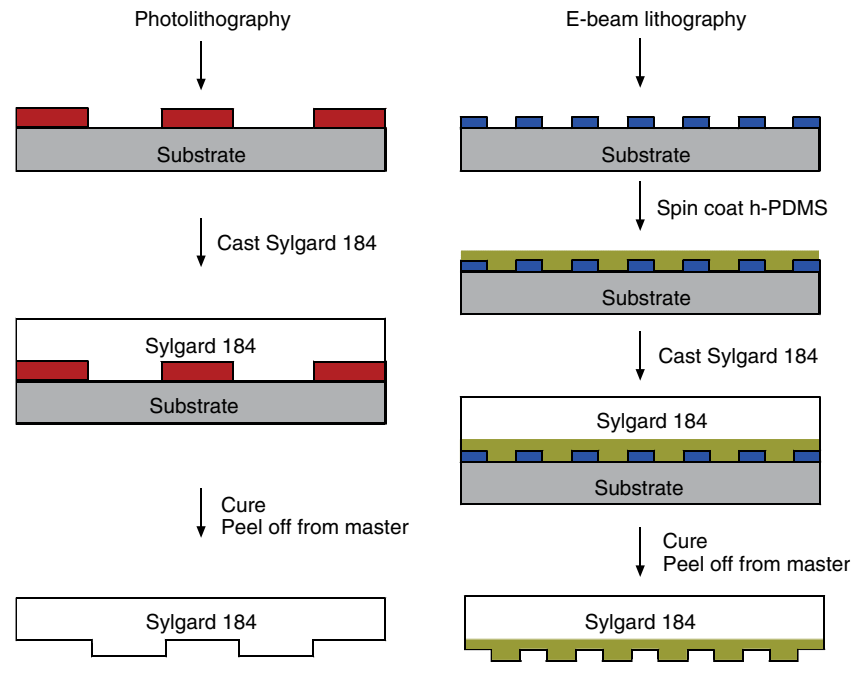

Figure 2 | Schematic illustration of the procedures for fabricating normal PDMS (left panel) and h-/PDMS (right panel) stamps, respectively. The addition of a thin layer of hard PDMS allows one to work with features $<500 \mathrm{~nm}$ in lateral dimensions.

stamps from masters with relatively low relief structures. In this study, the 'hard' PDMS or h-PDMS is based on vinyl and hydrosilane end-linked polymers with a higher modulus $\left(\sim 9 \mathrm{~N} / \mathrm{mm}^{2}\right)$ than that of Sylgard 184 PDMS $\left(\sim 2 \mathrm{~N} / \mathrm{mm}^{2}\right)$.

$\mu \mathrm{CP}$. There are three major steps involved in $\mu \mathrm{CP}$ (see Fig. 1), by which an 'ink'-typically an alkanethiol (a compound containing an - SH group)-is transferred from PDMS to a surface defined by a thin film of gold or silver supported on a silicon wafer or glass slide. Conformal, molecular/atomic-level contact between a PDMS stamp and the surface of a substrate is the key to successful transfer of ink molecules from the stamp to the substrate. $\mu \mathrm{CP}$ is widely used in printing alkanethiols on thin films of $\mathrm{Au}^{10}, \mathrm{Ag}^{45}, \mathrm{Pd}^{46}$ and $\mathrm{Pt}^{47}$, and to a smaller extent (and with more difficulties) of alkylsiloxanes on silicon/silicon dioxide or glass ${ }^{48}$. The ink molecules form SAMs on these surfaces during the printing process. The surface properties of a SAM can be controlled by modifying the end group (the functional group distal to the surface); a major use of $\mu \mathrm{CP}$ is to pattern organic groups useful in molecular recognition of biomolecules or of adhesive proteins for use in the cell biology $y^{49}$. On the basis of a comparison study, printed SAMs on Pd are operationally more stable for applications in cell biology than those formed on $\mathrm{Au}$ or $\mathrm{Ag}^{50}$. $\mu \mathrm{CP}$ has also been used to directly pattern arrays of proteins on silicon or glass substrates (see Fig. 1) $)^{51}$. Furthermore, $\mu \mathrm{CP}$ (with a stamp fabricated in gels rather than PDMS) can be used to directly print bacterial ${ }^{52}$ or mammalian cells ${ }^{53}$.

REM or embossing of structures in a polymer. REM consists of three steps (see Fig. 1) ${ }^{11}$ : (i) creating a topographically patterned master; (ii) transferring the pattern on the master into PDMS by REM; and (iii) transferring the pattern on the PDMS back into a replica of the original master by solidifying a liquid prepolymer such as UV-curable polyurethane (PU), or thermally curable epoxy, against the PDMS mold. REM offers a number of advantages over traditional fabrication process at the nanoscale such as EBL: 
(i) REM can produce many copies $(>50)$ of molds, replicas and patterned surfaces from each master with a possible resolution at the atomic level ${ }^{54}$; (ii) REM can work with a wide range of polymers other than e-beam-sensitive materials; (iii) REM allows patterning over large areas rapidly, not limited to a serial process defined by EBL; and (iv) REM can be applied to transfer patterns to nonplanar surfaces ${ }^{55}$.

SAMIM $^{14}$ uses an elastomeric mold, a polymer film and a solvent that dissolves in and softens the film to emboss pattern in the film (see Fig. 1). Typically, a PDMS mold is wetted with a liquid that is a good solvent for the polymer, and is brought into contact with the surface of the polymer film. The solvent dissolves a thin layer of the polymer at the surface, and the resultant fluid comprising polymer and solvent conforms to the surface topology of the mold. Although the mold is maintained in conformal contact with the substrate, the polymer solidifies as the solvent dissipates by entering into the PDMS and by evaporation to form relief structures with a pattern complementary to that on the surface of the PDMS mold. SAMIM forms patterned structures in a polymer under ambient conditions with soft molds rather than at elevated temperatures with rigid molds as required for conventional embossing ${ }^{56}$.

\section{MATERIALS}

\section{REAGENTS}

- Photoresists. As two examples of commonly used photoresists, positive AZ resists cover the range of thickness from 0.5 to $50 \mu \mathrm{m}$ (MicroChemicals $\mathrm{GmbH}$ ) and negative SU-8 2000 resists can be used with thickness ranging from 10 to $100 \mu \mathrm{m}$ (MicroChem). When choosing a photoresist (positive versus negative), one needs to consider the following factors: (i) the cost and the easiness to work with, (ii) the range of film thicknesses, (iii) the aspect ratio of the patterned structures and (iv) if the resist structures need to be stripped off or not afterward. The information can be obtained from the vendors and a decision can be easily made by talking to the staff in charge of the clean room facility

- Electron-beam resists. Poly(methyl methacrylate) (PMMA) is the most popular, positive-tone resist (MicroChem). It has a resolution close to $20 \mathrm{~nm}$ in lateral dimensions and conveniently accessible thickness (vertical dimension) in the range of 100-1,000 nm. SU-8 2005 and SU-8 2002 are two negative-tone e-beam resists (MicroChem), with a resolution of $100 \mathrm{~nm}$ for lateral dimensions and a range of accessible thicknesses from 100 to $500 \mathrm{~nm}$

- Tridecafluoro-1,1,2,2-tetrahydrooctyl-1-trichlorosilane (TFOCS, United Chemical Technology, cat. no. 6H-9283) ! CAUTION It is corrosive and toxic, avoid direct contact and always handle it in the fume hood.

- (7-8\% Vinylmethylsiloxane)-(dimethylsiloxane) copolymer (Gelest, cat. no. 6I-9467)

-1,3,5,7-Tetramethylcyclotetrasiloxane (Gelest, cat. no. 4E-4963)

- Platinum divinyltetramethyldisiloxane (Gelest, cat. no. 6H-92)

- (25-30\% Methylhydrosiloxane)-(dimethylsiloxane) copolymer (Gelest, cat. no. 6D-8641) $\Delta$ CRITICAL Store this copolymer in a refrigerator.

- Sylgard 184 silicone elastomer base and Sylgard 184 elastomer curing agent (Dow Corning)

- Hexadecanethiol (HDT, >99\%, Aldrich, cat. no. 2917-26-2)

- Green- and red-labeled chicken anti-goat IgG antibodies (Alexa Fluor 488 and Alexa Fluor 594, Invitrogen)

- Bovine serum albumin (BSA (Fraction V), Calbiochem)

- UV-curable PU (NOA 73, Norland Products)

- Thermally curable epoxy (TRA BOND BA F113, TRA-CON)

- PE-60 polyethylene tubing (Model No. BPE-T20, Instech Laboratories)

- Gold or silver substrates are typically prepared by thermal or e-beam evaporation. They can also be obtained from commercial vendors (e.g., Sigma-Aldrich, cat. no. 643262). Immediately before use, the commercial substrates should be cleaned using a cleaning solution (e.g., Sigma-Aldrich, cat. no. 667978) for 30-60 s to remove organic materials that tend to adsorb on the metal surface during shipping and storage. Cleaning is also recommended for substrates that have been stored (typically in a Petri dish and sealed with Parafilm) for more than 1 week.

- Ferricyanide etching solution for thin films of $\operatorname{gold}^{57} . \mathrm{K}_{2} \mathrm{~S}_{2} \mathrm{O}_{3}(0.1 \mathrm{M})$, $\mathrm{K}_{3} \mathrm{Fe}(\mathrm{CN})_{6}(0.01 \mathrm{M}), \mathrm{K}_{4} \mathrm{Fe}(\mathrm{CN})_{6}(0.001 \mathrm{M})$ and $\mathrm{KOH}(1 \mathrm{M})$ in water $\triangle$ CRITICAL The ferricyanide compound is sensitive to light, and the solution must be freshly prepared each time and used within $6 \mathrm{~h}$. The cyanide group can be released as hydrogen cyanide ( $\mathrm{HCN}$, highly toxic!) when ferricyanide is mixed with a strong acid. The etching solution should be treated as a hazardous waste and disposed according to the EH\&S regulations.

- Ferricyanide etching solution for thin films of $\operatorname{silver}^{57} \cdot \mathrm{K}_{2} \mathrm{~S}_{2} \mathrm{O}_{3}(0.1 \mathrm{M})$, $\mathrm{K}_{3} \mathrm{Fe}(\mathrm{CN})(0.01 \mathrm{M})$ and $\mathrm{K}_{4} \mathrm{Fe}(\mathrm{CN})(0.001 \mathrm{M})$ in water. See the information about the etching solution for gold thin films.

- Acetone (Sigma-Aldrich, cat. no. 534064)

- Ethanol (Sigma-Aldrich, cat. no. 459836)

- Anisole (Sigma-Aldrich, cat. no. 296295)

EQUIPMENT

- Spin coater (for spin coating resists as thin films, e.g., SPI Supplies, Model KW-4A)

- UV mask aligner (for photolithography, e.g., Karl Suss, Mask Aligner MJB3)

- Electron-beam writer (for e-beam lithography, e.g., Leica VB 6-HR, Leica or JEOL JBX 9300FS, JEOL)

- Dessicator (for surface modification, e.g., Sigma-Aldrich, cat. no. Z119008)

- Vacuum line (for surface modification and removal of bubbles)

- Hotplates (for baking resist films, e.g., Sigma-Aldrich, cat. no. CLS6795200)

- Nitrogen gas line (for drying stamps and substrates)

- Oxygen plasma cleaner (for surface modification and removal of residual polymer film, e.g., PDC-001, Harrick Scientific)

- UV light source for curing PU (Model B-100A, UVP)

- Thermal or electron-beam evaporator (for preparing supported thin films of gold or silver, e.g., Edwards 306)

- Optical microscope (for characterizing patterns on masters and stamps at the microscale, e.g., Zeiss AXIO IMAGER A1m, Zeiss)

- Scanning electron microscope (SEM, for characterizing patterns on masters and stamps at both micro- and nanoscale, e.g., Nova NanoSEM 230 , FEI)

- Atomic force microscope (AFM, for characterizing patterns on masters and stamps at both micro- and nanoscale, e.g., NanoMan, Veeco). Note that SEM and AFM are typically hosted in a user facility on the campus and one has to be trained by the staff before having access to any one of these instruments

\section{PROCEDURE}

\section{Design of the pattern}

1| Use a CAD software to design the pattern. It takes $2-4 \mathrm{~h}$ to get familiar with Freehand or Illustrator software while it may take up a week or two to get trained on the basics of AutoCAD.

$\triangle$ CRITICAL STEP When using Freehand or Illustrator, set the canvas to the size of the printed page so that the features in the pattern will not change their dimensions during the printing process. 
Fabrication of the photomask

2| Fabricate transparency masks (with feature sizes larger than $20 \mu \mathrm{m}$ ) using high-resolution printing or order chrome photomasks from commercial suppliers. The turnaround time for transparency printing service is around a few days while it may take a month for delivery of a chrome mask after the design is finalized.

\section{Fabrication of the master TIMING 2-4 $\mathrm{h}$}

3| Determine the relief height ( $H$ in Fig. 3 ) on the master by optimizing the aspect ratios- $H / L$ and $H / D$ - to prevent pairing and sagging of the elastomeric stamp. This information serves as a guide to select the range of thickness for the photoresist or e-beam resist films.

$\triangle$ CRITICAL Although the elastomeric character is essential for a conformal contact between the stamp and substrate required for contact printing, transfer molding and decal transfer, it can become a source of technical problems (e.g., lateral collapse or pairing of narrowly spaced features or sagging of the region between two widely separated features) in some designs (see Fig. 3$)^{58}$. Also, use a 111 rather than 100 silicon wafer to fabricate the master. Otherwise, the master can be easily broken during the preparation of a PDMS stamp.

\section{$?$ TROUBLESHOOTING}

4| Perform photolithography to fabricate a master with patterned AZ positive-tone photoresist or SU-8 negative-tone photoresist on the surface. A typical process takes about 2-4 $\mathrm{h}$.

$\triangle$ CRITICAL STEP To achieve the best result, users have to follow the specific protocols that have been optimized for spin coating, UV exposure and film developing-the three major steps of a typical photolithography process (for details, refer to the information offered by the commercial supplier of the photoresist and the resource at Cornell
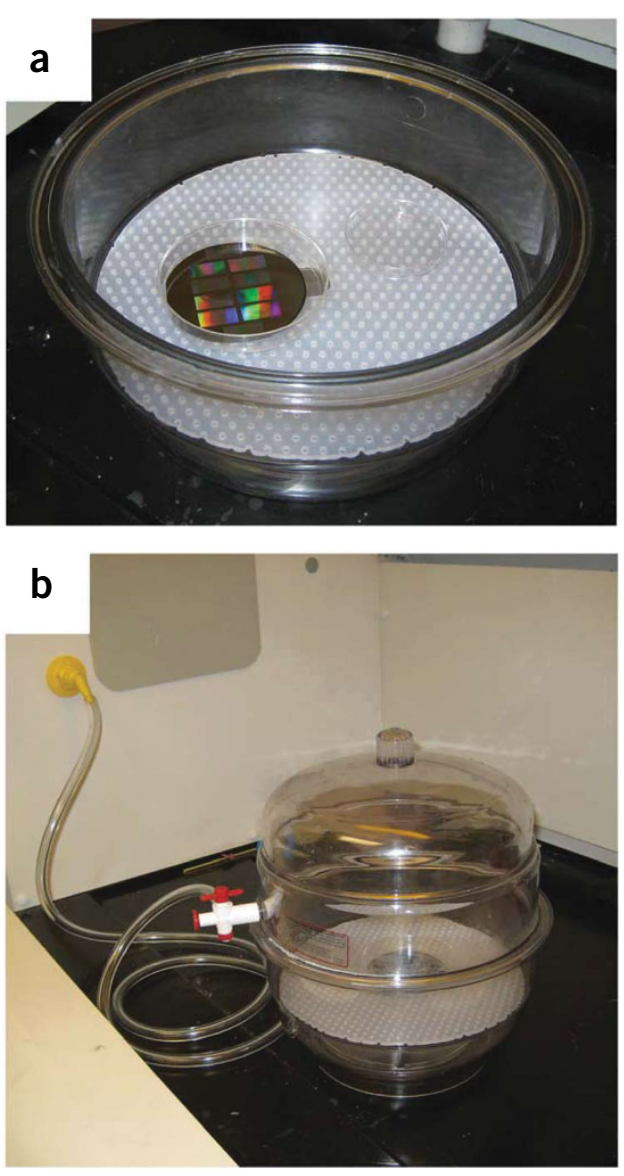

a

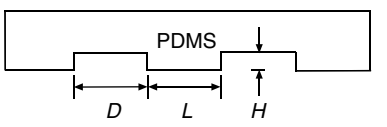

(Optimal aspect ratio: $0.5<H / L<5, H / D>0.05$ )

b

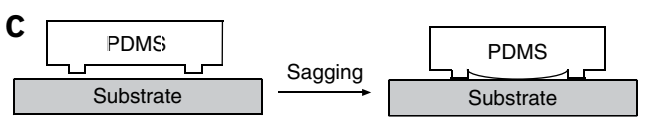

Figure 3 | Schematic illustrations of a PDMS stamp (a) and two possible problems that may arise from the softness of an elastomer: (b) lateral collapse of relief structures (or commonly known as 'pairing') with aspect ratios $H / L>5$; and (c) sagging of recessed structures with aspect ratios $H / L<0.5$.

\section{NanoScale Science \& Technology Facility).}

5| Perform EBL using an SEM system or a dedicated e-beam writer to generate a master in PMMA (positive tone) or SU-8 (negative tone). The writing process may take hours depending on the dose (or exposure time), the size of writing field and the density of writing objects. It requires extensive training (at least 8-10 h) in use of an SEM and a writing program. For detailed procedure, refer to the EBL resource at Cornell NanoScale Science \& Technology Facility.

\section{Fabrication of Sylgard 184 PDMS stamp TIMING 6-8 h}

6| Place the master in a Petri dish and then in a dessicator, together with a smaller Petri dish containing a few drops of TFOCS (Fig. 4). Connect the dessicator to the vacuum line of the fume hood and evacuate the air from the dessicator-this process takes about $30 \mathrm{~min}$, which allows TFOCS to vaporize and deposit as a monolayer on the master through siloxane bonding. Be sure to place the dessicator in the fume hood, as TFOCS is corrosive and toxic. The TFOCS vapor should transform the surface of a master from hydrophilic to hydrophobic; this modification

Figure 4 | Photographs of the dessicator used to treat the surface of the master with TFOCS vapor. $(\mathbf{a}, \mathbf{b})$ A few drops of TFOCS liquid were placed in the smaller Petri dish (a) before the dessicator was capped and connected to the vacuum line (b). Because the master contained periodic arrays of lines and dots $5 \mu \mathrm{m}$ in size, one can clearly see the diffraction colors from the surface. 
prevents the cured PDMS from sticking to the master. This step is required for a newly fabricated master. Once the modification is done, the master can be used to fabricate multiple copies (at least 20) of PDMS stamps without additional surface treatment.

\section{? TROUBLESHOOTING}

7| Take a common plastic cup (e.g., a Chinet 9-oz cut crystal plastic cup) and fill it with 10 parts of Sylgard 184 base and 1 part of the curing agent by weight. Use a balance to carry out weighing. When a Petri dish of $75 \mathrm{~mm}$ in diameter is used, one needs to weigh $70 \mathrm{~g}$ of the base and $7 \mathrm{~g}$ of the curing agent (10:1 ratio) in order to obtain PDMS stamps of roughly 10-mm thickness.

$\triangle$ CRITICAL STEP Sylgard 184 is a thermal curable elastomer, which comes as a two-component kit consisting of the base and the curing agent. It is not necessary to weigh the base and curing agent with accuracy $>5 \%$, as a small variation in the amount of either component will not affect the performance of resultant PDMS stamps. The mass ratio can be controlled to adjust the softness of cured elastomer-e.g., 5:1 for stiffer PDMS and 20:1 for softer PDMS. It is preferable to use nitrile or power-free latex gloves when handling the PDMS prepolymer. It has been suspected that the powder in typical latex gloves may contain a material that can inhibit the curing process.

8| Use a plastic spoon or a few sticks of wood Q-tips (Cotton-Tiped Applicator of 6") to thoroughly mix the base and curing agent for at least $3 \mathrm{~min}$. Air bubbles are generated in the mixing process but they can be easily removed in the next step.

$\triangle$ CRITICAL STEP Ensure that the two components are completely mixed. Incomplete mixing may affect the curing behavior, homogeneity and mechanical properties of the resultant PDMS.

9| Place the master on a Petri dish and pour the liquid mixture (with trapped bubbles) to the desired thickness; remove the bubbles by placing the Petri dish in a dessicator and connect to the vacuum line of the fume hood for 15 min or until most of the bubbles disappear; and turn off the vacuum and vent the dessicator.

$\triangle$ CRITICAL STEP It is important to complete this step after pouring the mixed PDMS onto the master so that no bubbles will be trapped at the interface between the master and PDMS. Also, ensure to eliminate all the bubbles trapped in the PDMS liquid mixture before proceeding to the next step-thermal curing.

10| Place the Petri dish containing both the master and PDMS prepolymer in an oven set to $70{ }^{\circ} \mathrm{C}$ and cure it for $6 \mathrm{~h}$. Figure 5a shows a photograph of the cured PDMS. The PDMS can also be cured at room temperature $\left(20^{\circ} \mathrm{C}\right)$, but it may take up to $48 \mathrm{~h}$ and the product tends to be softer than the sample cured at an elevated temperature. The curing takes place faster as the temperature increases.

11| Break the side of the Petri dish with a plier and separate the block of PDMS/master from the Petri dish carefully; use a scalpel or razor blade to remove the thin layer of PDMS on the backside of the master; and carefully peel the PDMS stamp off the master (Fig. 5b,c). With the patterned side facing up, use a razor blade or scalpel to cut the PDMS disk into stamps with the desired dimensions for different applications (Fig. 5d).
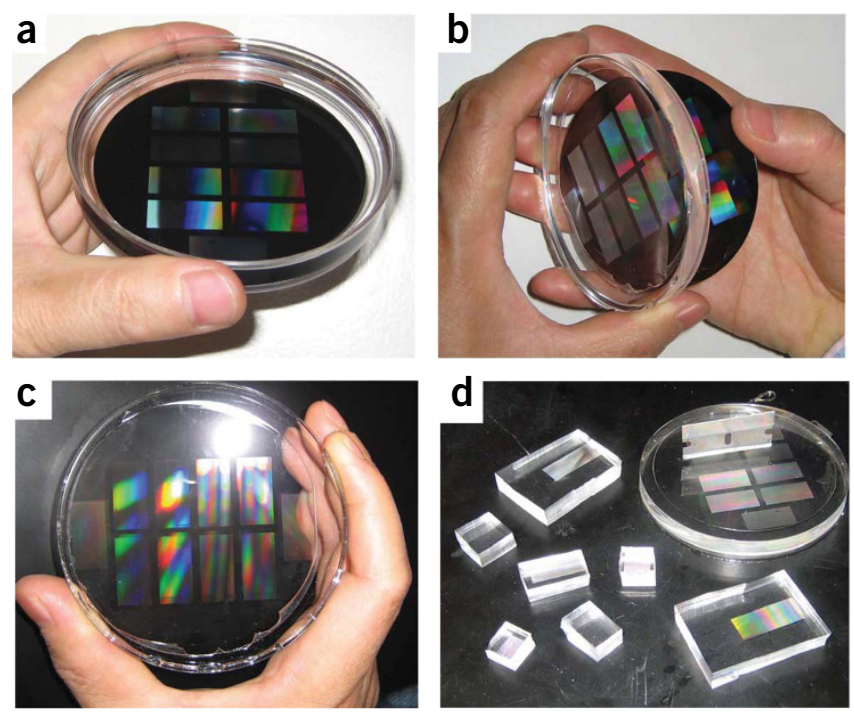

PAUSE POINT If the stamp contains periodic structures with lateral dimensions in the range of 1-10 $\mu \mathrm{m}$, one can quickly examine the quality of the stamp by viewing the diffraction color from an angle (Fig. 5c). One can typically use the same master to fabricate $\geq 20$ PDMS stamps. Keep the master in a covered Petri dish or other containers to avoid contamination of its surface by dust particles.

Figure 5 | Photographs of four major stages involved in the fabrication of PDMS stamps. (a-d) (a) Curing a liquid prepolymer to PDMS on top of the master placed on a Petri dish. (b) Separating the cured PDMS block from the master. (c) Viewing the diffraction colors from an oblique angle. (d) Cutting the PDMS block into smaller pieces (stamps) using a razor blade. When the pattern contains periodic structures with feature sizes in the range of 1-10 $\mu \mathrm{m}$, one can easily see diffraction colors (as shown in a-c) from the surface of the master or stamp. Although all the materials shown in this figure are not toxic, one might want to wear a pair of powder-free gloves for protection as required by the safety regulations set for a chemical laboratory. 


\section{Fabrication of h-/184 PDMS composite stamp TIMING 6-8 h}

12| Follow Steps 7 and 8 to prepare a mixture of $50 \mathrm{~g}$ of Sylgard 184 base and $5 \mathrm{~g}$ of the curing agent.

13| Use a stick of wood Q-tip to mix $3.4 \mathrm{~g}$ of (7-8\% vinylmethylsiloxane)-(dimethylsiloxane) copolymer with two drops of 1,3,5,7-tetramethylcyclotetrasiloxane and one drop of platinum divinyltetramethyldisiloxane in a Petri dish (55 mm in diameter). This amount of material is adequate to coat a master with a surface area of $1 \mathrm{~cm}^{2}$.

14| Remove bubbles by placing the Petri dish in a dessicator and connect to the vacuum line of the fume hood for 5 min. Turn off the vacuum, vent the dessicator and take out the Petri dish.

15 While the bubbles are being removed in the previous step, adjust the parameters of a spin coater to 500 r.p.m. for $5 \mathrm{~s}$, followed by 1,000 r.p.m. for 40 s.

$\triangle$ CRITICAL STEP It is important to set up the spin coater to a ready-to-run condition before proceeding to Step 16.

16| Add $1 \mathrm{~g}$ of (25-30\% methylhydrosiloxane)-(dimethylsiloxane) copolymer to the mixture in Step 14 and mix quickly by stirring with the same stick.

$\triangle$ CRITICAL STEP The new mixture will solidify within $5 \mathrm{~min}$, giving very little time to remove the bubbles as described in Step 14. Proceed to Step 17 immediately and the bubbles should be removed during the spin-coating process.

17| Load a TFOCS-modified master to the spin coater, pour the mixture prepared in Step 16 onto the master and turn on spinning immediately. If the mixture prepared in Step 16 has solidified, it is necessary to prepare a new mixture by repeating Steps $13-16$.

18| Place the coated master in a Petri dish (with the h-PDMS layer facing up) and bake it in an oven at $60{ }^{\circ} \mathrm{C}$ for 10 min.

19| Pour the mixture of Sylgard 184 prepared in Step 12 onto the h-PDMS layer, followed by Steps 9-11.

\section{$\mu \mathrm{CP}$ of HDT $\bigcirc$ TIMING $\mathbf{0 . 5 - 2} \mathrm{h}$}

20| Prepare gold or silver substrates using a thermal (or e-beam) evaporator that is often available in a conventional microfabrication facility. Typically, the thickness of the gold or silver film is controlled in the range of 20-100 nm, and there is an adhesion layer of titanium or chromium of 5-nm thickness between the gold or silver film and the silicon or glass support. The evaporation process often takes 1-2 h. Whenever possible, one should try to use freshly prepared substrates.

21| Prepare a HDT solution in ethanol ( 2 $\mathrm{mM})$.

22 Apply the HDT solution by swabbing the patterned side of a PDMS stamp with a cotton Q-tip for $5 \mathrm{~s}$ (the exact amount being transferred is not critical, as the ethanol will evaporate, and the HDT can diffuse into the PDMS) and dry the stamp in a stream of nitrogen for $30 \mathrm{~s}$.

PAUSE POINT To handle the stamp easily with fingers, the thickness of the stamp should be set to the range of $0.5-1.0 \mathrm{~cm}$.

23| Bring the PDMS stamp into contact with the gold or silver surface for 5-10 s (Fig. 6, left and middle panels). $\triangle$ CRITICAL STEP Avoid trapping air bubbles between the stamp and the substrate by initiating physical contact between these two surfaces from the edge with an angle.

$\triangle$ CRITICAL STEP It is critical to leave the stamp and the substrate in contact for $<20 \mathrm{~s}$, otherwise the thiol molecules may also be transferred from the recessed region of the stamp to the substrate through diffusion and thus ruin the pattern ${ }^{59}$. This is particularly important when alkanethiols with relatively short chains are used as the ink molecules. In addition, the thiol molecules may spread out from the edge of a raised region on the stamp and thus increase the size of the printed feature ${ }^{59}$.

24| Separate the PDMS stamp from the substrate without dragging (Fig. 6, right panel).

PAUSE POINT If needed for other experiments, one can fill the bare regions of the surface with a second SAM simply by dipping the printed substrate into an ethanol solution $(\sim 2 \mathrm{mM})$ of another alkanethiol terminated in a different end group for a period of 1-2 min. (Note that longer immersion time may cause displacement between the SAMs.) This strategy is useful when one has to pattern an alkanethiol (e.g., 16-mercaptohexadecanoic acid) that may crystallize during the printing process ${ }^{60}$. 
Figure 6 | Photographs of three major steps involved in $\mu \mathrm{CP}$ of alkanethiol on gold, which is evaporated as a thin film on a silicon substrate with 5-10 nm titanium (for e-beam evaporation) or chromium (for e-beam or thermal evaporation) as the adhesion layer: left, initiating contact between the PDMS stamp and the gold surface from the edge with an angle to avoid trapping air bubbles between them; middle, leaving the stamp in contact with gold for $10 \mathrm{~s}$; and right, separating the stamp from the gold surface. This figure is meant for illustration purpose only. In practice, one should wear a pair of powder-free gloves for protection as required by the safety regulations set for a chemical laboratory.
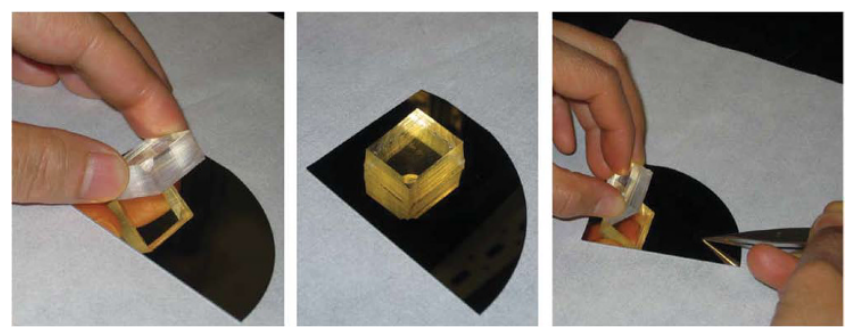

25 Immerse the substrate patterned with HDT monolayers in a ferricyanide etching solution to selectively remove the unprotected region of gold or silver under magnetic stirring (300 r.p.m.) at room temperature ${ }^{57}$. For silver films of 20-nm thickness, the etching time is around $7 \mathrm{~min}$. Figure 7 (top panel) shows SEM image of a typical example-an array of silver disks of $2.5 \mu \mathrm{m}$ in diameter and separated by $2.5 \mu \mathrm{m}$.

\section{$\mu \mathrm{CP}$ of proteins $\bigcirc$ TIMING $1 \mathrm{~h}$}

26| Prepare an IgG solution with a concentration in the range of $50-100 \mu \mathrm{g} \mathrm{ml}^{-1}$ in phosphate buffered saline (PBS).

27| Place a PDMS stamp (with the patterned surface facing up) in an oxygen plasma cleaner for oxidation at 200 mtorr of oxygen gas and $40 \mathrm{~W}$ of power for $1 \mathrm{~min}$. This treatment generates silanol group ( $\mathrm{Si}-\mathrm{OH}$ ) on the surface of the PDMS replica owing to oxidation ${ }^{34}$.
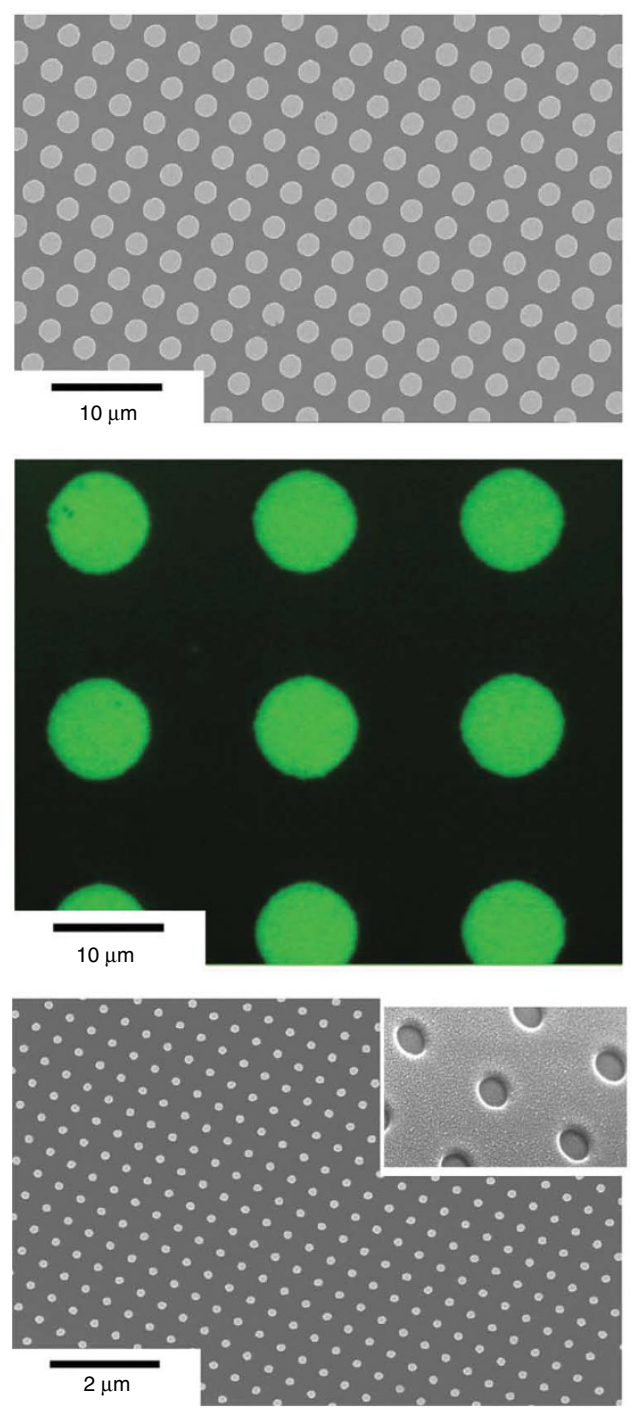

28| Immerse the plasma-treated PDMS stamp into the IgG solution, incubate for $45 \mathrm{~min}$ at room temperature and then remove it.

29| Rinse the PDMS stamp with PBS once and DI water thrice, and then dry the stamp under a stream of nitrogen for $30 \mathrm{~s}$.

30| Follow Steps 23 and 24, except use a glass slide as the substrate. Figure 7 (middle panel) shows an optical fluorescence micrograph of a typical example - an array of green-labeled IgG dots of $10 \mu \mathrm{m}$ in size. The protein was probably denatured to an uncharacterized extent.

$\Delta$ CRITICAL STEP Clean the glass substrate before use (see Step 20). Use a fresh stamp each time to achieve well-resolved patterns. When having problems to directly print a specific protein, one can use a BSA solution in PBS $\left(100 \mu \mathrm{g} \mathrm{ml}^{-1}\right)$ as the 'ink' and print features of BSA on a glass substrate, followed by filling the bare regions between the BSA features with that specific protein (by solution dipping). There may be some exchange and/or mixing between the printed BSA and the second protein.

\section{Fabrication of microfluidic devices in PDMS by REM TIMING 1-2 h}

31| Use photolithography to fabricate a master (Step 4), with a photomask printed on a transparency film.

Figure 7 | Three typical examples of micro- and nanostructures fabricated using soft lithography. Top, SEM image of an array of silver disks fabricated using $\mu \mathrm{CP}$ of HDT, followed by selective wet etching in a ferricyanide solution. Middle, fluorescence optical micrograph of an array of IgG dots fabricated using $\mu \mathrm{CP}$. Bottom, SEM image of an array of gold disks ( $100 \mathrm{~nm}$ in diameter) fabricated using SAMIM, followed by gold evaporation and lift-off. The inset shows SEM image of the pattern in PMMA fabricated using SAMIM. 
32| Make a PDMS replica of the master in Sylgard 184 (Steps 7-11).

33| Use a Harris Micro-Punch (Ted Pella, cat. no. 15091) to punch holes in PDMS that will serve as 'inlets' and 'outlets' for introducing and recovering fluids (e.g., samples, reagents and buffers), respectively. The holes punched in PDMS should be slightly smaller than the outer diameter of the polyethylene tube (see Step 37). When the tube is inserted, it exerts pressure on the PDMS and makes a waterproof seal. The tube can be removed and replaced with minimal effort.

34| Place the PDMS replica (with the patterned surface facing up) and a silicon or glass substrate in an oxygen plasma cleaner for oxidation at 200 mtorr of oxygen gas and $40 \mathrm{~W}$ of power for 1 min. Surface-oxidized PDMS can seal to PDMS, glass, silicon, polystyrene, polyethylene or silicon nitride, providing that these surfaces have also been exposed to oxygen plasma.

35| Place the side of the PDMS patterned with channels on the silicon or glass substrate to form a conformal contact.

36| Place the device in an oven at $60^{\circ} \mathrm{C}$ for $1 \mathrm{~h}$ to strengthen the adhesive seal (probably by dehydration: $\mathrm{Si}-\mathrm{OH}+\mathrm{HO}-\mathrm{Si} \rightarrow \mathrm{Si}-\mathrm{O}-\mathrm{Si}+\mathrm{H}_{2} \mathrm{O}$ ).

37| Insert PE-60 polyethylene tubing into the inlet(s) and outlet(s). The other end of the inlet tube is then connected to a 21-gauge syringe needle for sample injection. This procedure allows one to couple syringes and syringe pumps to microfluidic devices.

\section{Fabrication of PU or epoxy nanostructures by REM $\bigcirc$ TIMING 1-2 $h$}

38| Use EBL to fabricate a master (Step 5).

39| Fabricate an h-/184 PDMS composite stamp (or mold, in the case of molding rather than printing) following Steps $12-19$.

$\triangle$ CRITICAL STEP To facilitate separation in Step 44, control the total thickness of the PDMS mold in the range of $0.2-0.5 \mathrm{~cm}$.

40| Place a drop of UV-curable PU or thermally curable epoxy on a glass or silicon substrate. Mix the epoxy prepolymer and the curing agent thoroughly and place the mixture in a dessicator to eliminate air bubbles by connecting to the vacuum line of the fume hood. In general, prepolymers with high molecular weights are reasonably insoluble in PDMS. However, small compounds such as monomers and curing agents tend to diffuse into PDMS, causing the cured product to stick to PDMS during the molding process.

41| Place the h-/184 PDMS mold on top of the drop of prepolymer (with the patterned side facing the liquid prepolymer) and press down with hand to form contact. If necessary, squeeze out air bubbles by tapping the PDMS mold with the flat head of tweezers.

42| For UV-curable PU, cure under UV light and at room temperature for $30 \mathrm{~min}$. For epoxy, cure at room temperature for $15 \mathrm{~min}$ and then in an oven at $60^{\circ} \mathrm{C}$ for $30 \mathrm{~min}$. Peel off the PDMS mold carefully. The PU or epoxy structures should remain on the supporting substrate, provided that the adhesion between them and the support is stronger than that between them and the PDMS.

$\triangle$ CRITICAL STEP If the curing time is too long, the PU or epoxy structures will stick to the PDMS mold and ruin the patterned surface. If it is too short, the prepolymer may have not been completely cured, which can leave behind some liquid prepolymer on both the PU replica and the PDMS mold.

\section{Fabrication of PMMA structures by SAMIM $\bigcirc$ TIMING 0.5-1 $\mathrm{h}$}

43| Prepare a 3\% (wt/wt) PMMA solution by diluting the commercial e-beam resist with anisole. Dispense some of this solution onto a silicon or glass substrate to cover roughly $75 \%$ of the surface, and spin coat at 4,000 r.p.m. for $40 \mathrm{~s}$ to generate a uniform film of 100-nm thickness.

44| Bake the PMMA-coated substrate on a hotplate at $180^{\circ} \mathrm{C}$ for $90 \mathrm{~s}$ to completely remove all the anisole trapped in the PMMA film. As anisole has a relatively high boiling point $\left(154^{\circ} \mathrm{C}\right)$, it tends to evaporate slowly, interfering with the molding process. 
45 Apply acetone ( $2 \mu \mathrm{l}$ per $1 \mathrm{~cm}^{2}$ of area) to the patterned surface of an $\mathrm{h}-/ 184$ PDMS mold and bring it into contact with the PMMA film for 5 min.

$\triangle$ CRITICAL STEP Acetone is a good solvent for PMMA. Acetone cannot, however, spread on a PDMS surface, and will stay as a droplet on the PDMS mold until the mold is brought into contact with the PMMA film.

46| Bake the PMMA/acetone/h-PDMS sample on a hotplate at $60^{\circ} \mathrm{C}$ for 5 min to remove acetone completely. The PMMA layer is dissolved by acetone on contact with the PDMS mold and the resultant (probably gel-like) fluid comprising PMMA and acetone conforms to the surface topography of the mold. While maintained in conformal contact with the substrate, the PMMA solidifies as acetone dissipates, forming relief structures with a pattern complementary to that on the surface of the mold.

47| Remove the PDMS stamp carefully to leave patterned PMMA nanostructures on the surface of the substrate.

$\triangle$ CRITICAL STEP SAMIM can generate patterned relief structures over a relative large area $\left(\mathrm{ca} .3 \mathrm{~cm}^{2}\right)$ within a short period of time ( $10 \mathrm{~min})$. However, this process often leaves behind a thin film of PMMA (the residual layer) between the raised features protruding from the substrate. This residual layer prevents the underlying substrate from being attacked by chemical etchants, and must be removed by $0_{2}$ plasma etching if further process such as substrate etching or deposition is needed. In this case, proceed to Step 48 to remove the residue PMMA.

48| Place the sample with PMMA replica in a plasma cleaner to etch the surface for 2 min at 150 mtorr of oxygen and $40 \mathrm{~W}$ of power.

\section{TIMING}

Step 1, Design of the pattern

Step 2, Fabrication of the photomark

Steps 3-5, Fabrication of the master: $2-4 \mathrm{~h}$

Steps 6-11, Fabrication of Sylgard 184 PDMS stamp: $6-8 \mathrm{~h}$

Steps 12-19, Fabrication of h-/184 PDMS composite stamp: $6-8 \mathrm{~h}$

Steps $20-25, \mu \mathrm{CP}$ of HDT: $0.5-2 \mathrm{~h}$

Steps $26-30, \mu \mathrm{CP}$ of proteins: $1 \mathrm{~h}$

Steps 31-37, Fabrication of microfuidic devices in PDMS by REM: $1-2 \mathrm{~h}$

Steps 38-42, Fabrication of PU or epoxy nanostructures by REM: 1-2 $\mathrm{h}$

Steps 43-48, Fabrication of PMMA structures by SAMIM: $0.5-1 \mathrm{~h}$

\section{? TROUBLESHOOTING}

\section{Step 3}

As illustrated in Figure 3, if the aspect ratio $(H / L)$ of the raised region is too high, the stress originating from gravity, adhesion and capillary force may cause the relief structures to collapse. Alternatively, if the aspect ratio $H / D$ is too small, the recessed region will not be able to retain its structure during stamping. These problems could be minimized by designing patterns having the aspect ratios between 0.5 and 5 for $H / L$, and $>0.05$ for $H / D$, respectively. The use of thin PDMS stamps mounted on rigid supports ${ }^{44}$, or designs having 'posts' (like the posts of a bridge spanning across a wide river) to prevent sagging ${ }^{61}$, also provides solutions to these problems in some cases.

\section{Step 6}

Failure often occurs when TFOCS is in use beyond its shelf life. Perform a hydrophobicity test on the master with a small drop of water. If the modification by TFOCS is successful, the water drop should not spread on the surface of the master. Before moving to the next step, completely remove any water that may result from the hydrophobicity test in a stream of nitrogen.

\section{ANTICIPATED RESULTS}

Upon completion of the experiments described in this protocol, one should obtain the following structures or devices: (i) patterned features of HDT on gold or silver substrates; (ii) patterned microstructures of gold or silver supported on silicon or glass substrates; (iii) a patterned array of IgG dots on a glass slide; (iv) a microfluidic device with both inslet and outlet incorporated; ( $v$ ) patterned nanostructures of PU, epoxy or PMMA supported on a silicon or glass substrate; and (vi) a patterned array of gold nanostructures supported on a silicon or glass substrate. Figure 7 shows three typical examples of micro- and nanostructures fabricated using soft lithography: (top panel) an SEM image of silver disks patterned using $\mu \mathrm{CP}$, followed by wet etching in an aqueous ferricyanide solution; (middle panel) a fluorescence optical micrograph of IgG dots directly printed on a glass slide with a PDMS stamp; and (bottom panel) an SEM image of gold disks obtained by lift-off with the PMMA template (see the inset) fabricated using SAMIM. 
ACKNOWLEDGMENTS Both Washington University and Harvard University are members of the National Nanotechnology Infrastructure Network (NNIN), which is supported by the National Science Foundation under award no. ECS- 0335765 .

AUTHOR CONTRIBUTIONS D.Q. is responsible for all the experiments described in this article and preparation of the paper; Y.X. is partially responsible for experimental planning and preparation of the paper; and G.M.W. is responsible for providing guidance for the experiments and for editing and proofreading the paper.

Published online at http://www.natureprotocols.com/.

Reprints and permissions information is available online at http://npg.nature.com/ reprintsandpermissions/.

1. Whitesides, G.M. The right size: nanobiotechnology. Nat. Biotech. 21, 1161-1165 (2003).

2. Beaudet, A.L. \& Belmont, J.W. Array-based DNA diagnostics: let the revolution begin. Annu. Rev. Med. 59, 113-129 (2008).

3. Zhang, Y. \& Ozdemir, P. Microfluidic DNA amplification-a review. Anal. Chim. Acta 638, 115-125 (2009).

4. Herold, K.E. \& Rasooly, A. (eds.). Lab-on-a-Chip Technology: Fabrication and Microfluidics. (Caister Academic Press, Hethersett, Norwich, UK, 2009).

5. Cui, H.F. et al. Microelectrode array biochip: tool for in vitro drug screening based on the detection of a drug effect on dopamine release from PC12 cells. Anal. Chem. 78, 6347-6355 (2006).

6. Li, N., Tourovskaia, A. \& Folch, A. Biology on a chip: microfabrication for studying the behavior of cultured cells. Crit. Rev. Biomed. Eng. 31, 423-488 (2003).

7. Moreau, W.M. Semiconductor Lithography: Principles and Materials (Plenum, New York, 1998).

8. Fodor, S.P. et al. Light-directed, spatially addressable parallel chemical synthesis. Science 251, 767-73 (1991).

9. Xia, Y. \& Whitesides, G.M. Soft lithography. Angew. Chem. Int. Ed. Engl. 37, 551-575 (1998).

10. Kumar, A. \& Whitesides, G.M. Features of gold having micrometer to centimeter dimensions can be formed through a combination of stamping with an elastomeric stamp and an alkanethiol ink followed by chemical etching. Appl. Phys. Lett. 63, 2002-2004 (1993).

11. Xia, Y. et al. Replica molding using polymeric materials: a practical step toward nanomanufacturing. Adv. Mater. 9, 147-149 (1997).

12. Zhao, X.-M., Xia, Y. \& Whitesides, G.M. Fabrication of three-dimensional microstructures: microtransfer molding. Adv. Mater. 8, 837-840 (1996).

13. Kim, E., Xia, Y. \& Whitesides, G.M. Polymeric microstructures formed by moulding in capillaries. Nature 376, 581-584 (1995).

14. Kim, E., Xia, Y., Zhao, X.-M. \& Whitesides, G.M. Solvent-assisted microcontact molding: a convenient method for fabricating threedimensional structures of polymeric materials. Adv. Mater. 9, 651-654 (1997).

15. Rogers, J.A., Paul, K.E., Jackman, R.J. \& Whitesides, G.M. Using an elastomeric phase mask for sub-100 nm photolithography in the optical near field. Appl. Phys. Lett. 70, 2658-2660 (1997).

16. Jeon, S. et al. Three-dimensional nanofabrication with rubber stamps and conformable photomasks. Adv. Mater. 16, 1369-1371 (2004).

17. Childs, W.R. \& Nuzzo, R.G. Decal transfer microlithography: a new softlithographic patterning method. J. Am. Chem. Soc. 124, 13583-13596 (2002).

18. Xu, Q., Rioux, R.M., Dickey, M.D. \& Whitesides, G.M. Nanoskiving: a new method to produce arrays of nanostructures. Acc. Chem. Res. 41 1566-1577 (2008).

19. Gates, B.D. et al. New approaches to nanofabrication: molding, printing, and other techniques. Chem. Rev. 105, 1171-1196 (2005).

20. Ginger, D.S., Zhang, H. \& Mirkin, C.A. Angew. Chem. Int. Ed. Engl. 43, 30-45 (2004).

21. Geissler, M. \& Xia, Y. Patterning: principles and some new developments. Adv. Mater. 16, 1249-1269 (2004).

22. Love, J.C. et al. Self-assembled monolayers of thiolates on metals as a form of nanotechnology. Chem. Rev. 105, 1103-1170 (2005).

23. George, J. Preparation of Thin Films (Marcel Dekker, New York, 1992).

24. Chen, C.S., Mrksich, M., Huang, S., Whitesides, G.M. \& Ingber, D.E. Geometric control of cell life and death. Science 276, 1425-1428 (1997).

25. von Philipsborn, A.C. et al. Microcontact printing of axon guidance molecules for generation of graded patterns. Nat. Protoc. 1, 1322-1328 (2006).
26. McDonald, J.C. et al. Fabrication of microfluidic systems in poly(dimethylsiloxane). Electrophoresis 21, 27-40 (2000).

27. Chung, K., Crane, M. \& Lu, H. Automated on-chip imaging and sorting of C. elegans. Nat. Protoc. 4 (2009); DOI: 10.1038/nprot.2009.105.

28. Sidorova, J.M., Li, N., Schwartz, D.C., Folch, A. \& Monnat, R.J. Microfluidic-assisted analysis of replicating DNA molecules. Nat. Protoc. 4, 849-861 (2009).

29. Ogunniyi, A.O., Story, C.M., Papa, E., Guillen, E. \& Love, J.C. Screening individual hybridomas by microengraving to discover monoclonal antibodies. Nat. Protoc. 4, 767-782 (2009).

30. Paguirigan, A.L. \& Beebe, D.J. Protocol for the fabrication of enzymatically crosslinked gelatin microchannels for microfluidic cell culture. Nat. Protoc. 2, 1782-1788 (2007).

31. Park, J.W., Vahidi, B., Taylor, A.M., Rhee, S.W. \& Jeon, N.L. Microfluidic culture platform for neuroscience research. Nat. Protoc. 1, 2128-2136 (2006).

32. Tourovskaia, A. \& Figueroa-Masot, X. \& Floch, A. Long-term microfluidic cultures of myotube microarrays for high-throughput focal simulation. Nat. Protoc. 1, 1092-1104 (2006).

33. Semlyen, J.A. \& Clarson, S.J. (eds). Silicone Polymers (Prentice-Hall, Englewood, New Jersey, 1993).

34. Hillborg, H. et al. Crosslinked polydimethylsiloxane exposed to oxygen plasma studied by neutron reflectometry and other surface specific techniques. Polymer 41, 6851-6863 (2000).

35. Whitesides, G.M. The origins and the future of microfluidics. Nature $\mathbf{4 4 2}$, 368-373 (2006).

36. Zheng, B., Tice, J.D. \& Ismagilov, R.F. Formation of arrayed droplets by soft lithography and two-phase fluidic flow, and application in protein crystallization. Adv. Mater. 16, 1365-1368 (2004).

37. Xia, Y., Rogers, J.A., Paul, K.E. \& Whitesides, G.M. Unconventional methods for fabricating and patterning nanostructures. Chem. Rev. 99, 1823-1848 (1999).

38. Anderson, B.B., Burgess, L.W. \& Brodsky, A.M. Grating light reflection spectroscopy of colloids and suspensions. Langmuir 13, 4273-4279 (1997).

39. Lee, K.-H. et al. Microfluidic systems integrated with two-dimensional surface plasmon resonance phase imaging systems for microarray immunoassay. Biosens. Bioelectron. 23, 466-472 (2007).

40. Stewart, M.E. et al. Quantitative multispectral biosensing and 1D imaging using quasi-3D plasmonic crystals. Proc. Natl. Acad. Sci. USA 103, 17143-17148 (2006).

41. Backofen, U., Matysik, F.-M. \& Lunte, C.E. A chip-based electrophoresis system with electrochemical detection and hydrodynamic injection. Anal. Chem. 74, 4054-4059 (2002).

42. Qin, D., Xia, Y. \& Whitesides, G.M. Rapid prototyping of complex structures with feature sizes larger than $20 \mu \mathrm{m}$. Adv. Mater. 8, 917-919 (1996).

43. Xia, Y., Tien, J., Qin, D. \& Whitesides, G.M. Non-photolithographic methods for fabrication of elastomeric stamps for use in microcontact printing. Langmuir 12, 4033-4038 (1996).

44. Odom, T.W. et al. Improved pattern transfer in soft lithography using composite stamps. Langmuir 18, 5314-5320 (2002).

45. Xia, Y., Kim, E. \& Whitesides, G.M. Microcontact printing of alkanethiols on silver and its application in microfabrication. J. Electrochem. Soc. 143, 1070-1079 (1996).

46. Love, J.C. et al. Self-assembled monolayers of alkanethiolates on palladium are good etch resists. J. Am. Chem. Soc. 124, 1576-1577 (2002).

47. Geissler, M., Chen, J. \& Xia, Y. Comparative study of monolayers selfassembled from octadecylisocyanide and octadecanethiol on polycrystalline Pt substrates. Langmuir 20, 6993-6997 (2004).

48. Xia, Y., Mrksich, M., Kim, E. \& Whitesides, G.M. Microcontact printing of octadecylsiloxane on the surface of silicon dioxide and its application in microfabrication. J. Am. Chem. Soc. 117, 9576-9577 (1995).

49. Mrksich, M. \& Whitesides, G.M. Using self-assembled monolayers to understand the interactions of man-made surfaces with proteins and cells. Ann. Rev. Biophys. Biomol. Struct. 25, 55-78 (1996).

50. Jiang, X., Bruzewicz, D.A., Thant, M.M. \& Whitesides, G.M. Palladium as a substrate for self-assembled monolayers used in biotechnology. Anal. Chem. 76, 6116-6121 (2004).

51. Bernard, A. et al. Printing patterns of proteins. Langmuir 14, 2225-2229 (1998).

52. Weibel, D.B. et al. A bacterial printing press that regenerates its ink: contact printing bacteria using hydrogel stamps. Langmuir 21, 6436-6442 (2005). 
PROTOCOL

53. Stevens, M.M. et al. Direct patterning of mammalian cells onto porous tissue engineering substrates using agarose stamps. Biomaterials $\mathbf{2 6}$, 7636-7641 (2005).

54. Gates, B.D. \& Whitesides, G.M. Replica molding of vertical features smaller than $2 \mathrm{~nm}$ by soft lithography. J. Am. Chem. Soc. 125, 14986-14987 (2003).

55. Xia, Y. et al. Complex optical surfaces by replica molding against elastomeric masters. Science 273, 347-349 (1996).

56. Chou, S.Y., Krauss, P.R. \& Renstrom, P.J. Imprint lithography with 25-nanometer resolution. Science 272, 85-87 (1996).

57. Xia, Y., Zhao, X.-M., Kim, E. \& Whitesides, G.M. A selective etching solution for use with patterned self-assembled monolayers of alkanethiolates on gold, silver and copper. Chem. Mater. 7, 2332-2337 (1995).
58. Delamarche, E., Schmid, H., Michel, B. \& Biebuyck, H. Stability of molded polydimethylsiloxane microstructures. Adv. Mater. 9, 741-746 (1997).

59. Xia, Y. \& Whitesides, G.M. Use of controlled reactive spreading of liquid alkanethiol on the surface of gold to modify the size of features produced by microcontact printing. J. Am. Chem. Soc. 117, 3274-3275 (1995).

60. Palacin, S. et al. Patterning with magnetic materials at the micron scale. Chem. Mater. 8, 1316-1325 (1996).

61. Biebuyck, H., Larsen, N.B., Delamarche, E. \& Michel, B. Lithography beyond light: macrocontact printing with monolayer resist. IBM J. Res. Develop. 41, 159-170 (1997). 